\title{
Extended - spectrum beta - lactamase E. coli and K. pneumoniae urinary tract infections
}

Prayong Vachvanichsanong ( $\Delta$ vprayong@gmail.com )

Prince of Songkla University https://orcid.org/0000-0003-3735-1771

\section{Edward B McNeil}

Prince of Songkla University

\section{Pornsak Dissaneewate}

Prince of Songkla University

\section{Research article}

Keywords: congenital anomalies of the kidney and urinary tract; extended-spectrum beta-lactamase Escherichia coli; extended-spectrum beta-lactamase Klebsiella pneumoniae, recurrent; urinary tract infection

Posted Date: August 26th, 2020

DOI: https://doi.org/10.21203/rs.3.rs-64451/v1

License: (c) (i) This work is licensed under a Creative Commons Attribution 4.0 International License. Read Full License 


\section{Extended-spectrum beta-lactamase $E$. coli and $K$. pneumoniae urinary tract infections}

Prayong Vachvanichsanong, M.D. ${ }^{1}$, Edward B McNeil, MSc. ${ }^{2}$, Pornsak Dissaneewate, M.D. ${ }^{1}$

${ }^{1}$ Department of Pediatrics, ${ }^{2}$ Epidemiology Unit, Faculty of Medicine, Prince of Songkla University, Hat Yai, Songkhla, THAILAND

\section{Corresponding author:}

Prayong Vachvanichsanong, M.D.

Department of Pediatrics, Faculty of Medicine, Prince of Songkla University, Hat Yai, Songkhla 90110, THAILAND

E-mail: vprayong@gmail.com

\section{E-mail address:}

Prayong Vachvanichsanong: vprayong@gmail.com

Edward B McNeil: edward.m@psu.ac.th

Pornsak Dissaneewate: pornsak.d@psu.ac.th

\section{Declarations}

Ethics approval: By Faculty of Medicine, Prince of Songkla University No. 071-361/41

Consent to participate: This article does not contain any studies with human participants or animals performed by any of the authors

$\square$ Consent for publication: The manuscript contains any individual person's data in any form

$\square \quad$ Availability of data and materials: Not applicable

$\square$ Competing interests: The authors declare that they have no competing interests

$\square \quad$ Funding: There is no funding resource

$\square \quad$ Authors' contributions

Dr. Prayong Vachvanichsanong conceptualized and designed the study, designed the data, collected data, carried out the initial analyses, drafted the initial manuscript, and reviewed and revised the manuscript.

Mr. Edward McNeil carried out the initial analyses, reviewed and revised the manuscript.

Dr. Pornsak Dissaneewate collected data, reviewed the manuscript Acknowledgements: Not applicable 


\section{Abstract}

Background: The prevalences of extended-spectrum beta-lactamase (ESBL) Escherichia coli (E. coli) and Klebsiella pneumoniae (K. pneumoniae) urinary tract infections (UTI) in children are increasing worldwide. We aimed to investigate the prevalence, clinical findings, impact and risk factors of ESBL E. coli / K. pneumoniae UTI.

Methods: The medical records of children with UTI aged < 15 years admitted to Prince of Songkla University Hospital were reviewed.

Results: Theirty-seven boys and 46 girls had ESBL in 102 UTI episodes; 85 boys and 103 girls had non-ESBL in all of their 222 UTI episodes. The median age at presentation was $1.5(0.7$ - 4.8) years for the ESBL group and $1.3(0.6$ - 3.9) for the non-ESBL group $(\mathrm{p}=0.2)$. Age and gender were not significantly different between the two groups. The prevalence of ESBL rose between 2004 and 2008 before plateauing at around 30-40\% per year. The prevalences in first and recurrent UTI were $27.3 \%$ and $46.5 \%$, respectively $(\mathrm{p}=0.003)$. Fever prior to UTI diagnosis was found in $78.4 \%$ of episodes in the non-ESBL group and $61.8 \%$ of episodes in the ESBL group $(\mathrm{p}=0.003)$. Multivariate analysis, children without fever $(\mathrm{OR}=2.14,95 \% \mathrm{CI}: 1.23-3.74)$ and those with recurrent UTI (OR=2.67, 95\% CI: 1.37 - 5.19) were more likely to have ESBL UTI. The presence of CAKUT had no effect on ESBL UTI.

Conclusions: ESBL was found in one-third of E. coli/K. pneumoniae UTI episodes. No clinical condition nor imaging study could predict ESBL. Recurrent UTI was the only independent risk factor.

KEYWORDS: congenital anomalies of the kidney and urinary tract; extended-spectrum beta-lactamase Escherichia coli; extended-spectrum beta-lactamase Klebsiella pneumoniae, recurrent; urinary tract infection 


\section{Background}

Escherichia coli (E. coli) and Klebsiella pneumoniae (K. pneumoniae) are the two most common causative organisms in childhood urinary tract infection (UTI) [1-5]. Both E. coli and K. pneumoniae are also the most common organisms that produce plasmid-mediated enzymes, also known as extended-spectrum betalactamase (ESBL), which results in beta-lactam antibiotic resistance including expanded spectrum cephalosporins and monobactams [5-10].

The increasing proportions of ESBL E. coli and $K$. Pneumoniae strains causing UTI in children is a concern worldwide due to empirical therapy failure which may result in more complicated consequences, such as sepsis, prolonged hospitalization and renal scarring, compared to their non-ESBL strains [5-13].

ESBL is commonly defined as ESBL infection is antibiotic resistance resulting in limited antibiotic options. Infections caused by ESBL E. coli and K. pneumoniae strains require parenteral antibiotic therapy. Ideally, sensitive antibiotics should be prescribed immediately when UTI is suspected; however, results of urine culture and antibiotic sensitivity take about 2-3 days to process so in practice, empirical antibiotics are prescribed without waiting for the sensitivity results. Empirical antibiotics are usually not sensitive to ESBL UTI but are still prescribed because ESBL-sensitive antibiotics are too broad to be prescribed initially in order to avoid drug resistance. The antibiotic may be changed 2-3 days later when the results of the urine culture are known. Thus the majority of ESBL UTI diagnoses and treatments are usually delayed.

In the modern world, with the increasing rates of antibiotic resistance, it is of the highest necessity to evaluate the characteristics and consequences of these infections in order to obtain early diagnosis and treatment to minimize the potential consequences. Additionally, the impact of ESBL UTI on antibiotic therapy needs to be determined as well as its association with congenital anomalies of the kidney and urinary tract (CAKUT). Knowing the differences in clinical conditions, urine findings and kidney and urinary tract imaging results between children with ESBL and non-ESBL UTI will be beneficial for antibiotic selection before the results of the urine culture are known.

\section{Objective}

To determine the prevalence of childhood ESBL E. coli and $K$. pneumoniae UTI and explore its impact and associated risk factors.

\section{Methods}

We retrospectively reviewed the medical records of children diagnosed with both nosocomial and community-acquired UTI aged less than 15 years admitted to Prince of Songkla University Hospital in southern Thailand from January 2004 to December 2013. Only children with single organism E. coli or K. pneumoniae strains were included in the study. UTI was confirmed by urine culture as follows: 1) 50,000 colonies $/ \mathrm{mm}^{3}$ or more on catheterized urine; 2) 100,000 colonies $/ \mathrm{mm}^{3}$ or more on clean catch midstream urine. Urine culture and antimicrobial susceptibility testing were performed by the hospital laboratory following standard guidelines.

Pyuria was defined as white blood cells $>10$ neutrophils per high-power field in urinalysis. Proteinuria was defined by a positive urine protein strip or quantitative urine protein to creatinine ratio $>0.2 \mathrm{mg} / \mathrm{mg}$. An abnormally high creatinine level was defined as any level above the upper normal range for age [14]. 


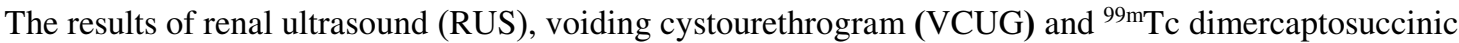
acid (DMSA) renal scans were obtained from routine reports. Hydronephrosis was determined by measuring the anteroposterior mid-renal pelvic diameter (APD) and graded as mild, moderate or severe according to the system used by the Society for Fetal Urology [15]. VCUG was performed in order to determine the anatomy of the lower urinary tract system, including the urinary bladder and urethra, particularly for diagnosis of vesicoureteral reflux, ureterocele, and/or posterior urethral valve. DMSA renal scans were performed according to standard clinical procedure and at least five months after confirmed UTI was evaluated. A normal DMSA scan is defined as no focal defect uptake of the tracer with an uptake difference between left and right kidneys of less than 10\%. An abnormal DMSA scan includes either renal scarring or dysplasia. Renal scarring is defined as a diffuse or sharp indentation in the kidney contour with thinning of the cortex. Dysplasia is defined as a differential renal function less than $35 \%$ [16].

The prevalence of ESBL-causing UTI, clinical findings, laboratory results and imaging studies were compared between the ESBL and non-ESBL UTI children.

\section{Statistical analysis}

The prevalences of UTI caused by ESBL and non-ESBL E. coli and K. pneumoniae bacteria strains were compared using the chi-squared test. Descriptive statistics are presented using medians and interquartile ranges for continuous variables and frequency and percentage for categorical variables. Comparisons of the proportions of ESBL vs non-ESBL UTI episodes across demographic and clinical factors were done using the rank-sum test or chi-squared test as appropriate. Independent risk factors of ESBL UTI were determined using multivariate logistic regression models. Statistical significance was considered as a p-value $<0.05$. R version 3.6.0 was used for all analyses [17].

\section{Results}

Demographic characteristics

Of 336 children diagnosed with 429 UTI episodes during the study period, E. coli and K. pneumoniae were causative in 271 children (80.7\%) and 324 episodes (75.5\%). The median (interquartile) age of the children at these episodes was 1.4 (0.6 - 4.1) years. Among the 271 children, 83 (37 boys, 46 girls) had 102 episodes in which at least one of their urine cultures was confirmed as an ESBL strain, and 188 children (85 boys, 103 girls) had 222 episodes in which all of the urine cultures were non-ESBL. Table 1 shows a comparison of the demographic and clinical factors between the ESBL and non-ESBL UTI episodes. The median (interquartile range) ages at presentation were $1.5(0.7$ - 4.8) years for the ESBL group and $1.3(0.6$ - 3.9) years for the nonESBL group $(\mathrm{p}=0.2)$. The proportions of ESBL infections among boys and girls were $30.3 \%$ and $32.6 \%$, respectively $(\mathrm{p}=0.7)$.

\section{Clinical findings and laboratory results}

The prevalence of ESBL in first UTI episodes was $27.3 \%$ compared to $46.5 \%$ in recurrent episodes $(\mathrm{p}=0.003)$ and among those with fever prior to UTI diagnosis was $26.6 \%$ compared to $44.8 \%$ among those without fever ( $\mathrm{p}=0.003)$. Fever was more common in younger (age <2 years) children $(79.5 \%)$ compared to older (age $>2$ years) children $(64.7 \%)(\mathrm{p}=0.003)$. The prevalences of ESBL among children with pyuria and proteinuria were $48.3 \%$ and $39.2 \%$, respectively, compared to $29.8 \%$ and $28.3 \%$ among those without these 
conditions (both $\mathrm{p}=0.07$ ). The prevalence of ESBL among children with abnormally high creatinine was $23.8 \%$ compared to $35.8 \%$ among those with normal creatinine $(p=0.2)$.

\section{Changes in ESBL prevalence over time}

Figure 1 shows the prevalences and proportions of ESBL and non ESBL UTI for each year of the study. The proportion of ESBL was $0 \%$ in 2004 but increased to over 30\% in 2008, thereafter it remained stable at around 30-40\% per year. ESBL E. coli was more common than ESBL K. pneumoniae but the prevalence of ESBL among $E$. coli strains $(70 / 265=26.4 \%)$ was lower than among $K$. pneumoniae strains $(32 / 59=54.2 \%$, $\mathrm{p}<0.001)$.

Antibiotics

Figure 2 shows the rates of resistance to various antibiotics among the 15 drugs tested, stratified by ESBL and non-ESBL. Children with ESBL UTI had a 100\% rate of resistance to cefuroxime, ceftazidime, and cefotaxime and a 99\% resistance rate to ampicillin and ceftriaxone. The resistance rates of children with nonESBL UTI to these antibiotics were lower ( $83 \%$ for ampicillin and $58 \%$ for co-trimoxazole but $<13 \%$ for the others). Multi-drug resistance was seen in all children with ESBL UTI and in 59\% with non-ESBL UTI. Only three drugs, namely meropenem, imipenem and colistin, had $0 \%$ resistance rates for all children.

Ceftriaxone was the most common antibiotic prescribed $(69.8 \%)$ in this study, followed by ertapenem $(7.4 \%)$ and ceftazidime $(6.8 \%)$.

Imaging study results

Table 2 shows a comparison of imaging study results and kidney abnormality findings between children with ESBL and non-ESBL UTI. The prevalences of ESBL UTI among those with abnormal RUS, VCUG and DMSA renal scan studies were $36.1 \%, 46.8 \%$ and $42.2 \%$, respectively, compared to $20.8 \%, 19.2 \%$ and $17.6 \%$ among those with normal imaging studies $(\mathrm{p}=0.018, \mathrm{p}<0.001, \mathrm{p}=0.016$, respectively).

Of the 271 children, 233 had at least one imaging study performed to diagnose CAKUT. The 38 children with no imaging study were significantly older (median age=5.1 years) compared to children with CAKUT (median age $=1.3$ years) and those with no CAKUT (median age $=11$ months).

CAKUTs were detected in 129 (47.6\%) children, of which 34.9\% had ESBL UTI, while the prevalence of ESBL UTI among those without CAKUT was $26.8 \%(\mathrm{p}=0.19)$. The most common congenital anomaly was primary vesicoureteral reflux (VUR) $(n=52)$, followed by isolated hydronephrosis $(n=43)$ and neurogenic bladder $(\mathrm{n}=18)$. Six children were found to have isolated renal damage without other kidney or bladder anomalies and all had non-ESBL UTI.

Table 3 shows the results of the multivariate analysis predicting ESBL E. coli/K. pneumoniae-caused UTI. The significant predictors were afebrility and recurrent UTI. Children with unknown CAKUT status were 4 times more likely have ESBL UTI than those with no CAKUT based on imaging studies. However, there was no difference in ESBL UTI between children with CAKUT compared to those without CAKUT. Afebrile UTI children $(\mathrm{OR}=2.14)$ and those with a recurrent $\mathrm{UTI}(\mathrm{OR}=2.67)$ were significantly more likely to have ESBL as a causative agent. 


\section{Discussion}

Childhood UTI in this group of Thai children shows that the prevalence of ESBL E. coli/K. pneumonia UTI increased in the decade between 2004 and 2013 but remained steady at around 30-40\% in the following 6 years. The proportion of $K$. pneumoniae ESBL was double that of E. coli ESBL. Neither age, gender, pyuria, proteinuria, abnormally high creatinine, or the presence of CAKUT could predict ESBL as a cause of UTI. However, a previous history of UTI and pre-diagnosis afebrility were significantly more common in the ESBL group. Older children had a significantly lower prevalence of fever $(\mathrm{p}=0.003)$ and although those with prediagnosis fever had a significantly lower prevalence of ESBL, age was not associated with ESBL.

The country-specific prevalence of ESBL E. coli/K. pneumoniae-caused UTI varies but has tended to increase over time [5-13].

A study from Egypt reported prevalences of ESBL in E. coli and K. pneumoniae of $41.9 \%$ and $48.8 \%$, respectively, and overall 43.5\% [4], a result similar to a study from Cambodia [5]. In a study conducted in 142 hospitalized Turkish children, the prevalence of ESBL was found to be as high as $49.3 \%, 49.1 \%$ of E. coli and $63.6 \%$ of K. Pneumonia [13]. The prevalence of ESBL in India among 1-month - 18-year-old patients ranged from $21.7 \%-33.2 \%$, with a higher prevalence in females [12] and in another study also from India the prevalence was $37.5 \%$, both results being similar to our study [18]. In two studies in recent years, one from Bangkok, Thailand and one from Korea, ESBL was found in around 20\% of childhood UTI patients [11, 19].

In one study in infants, age less than 1 year was reported to be an independent risk factor for ESBL UTI (OR=1.73, 95\% CI 1.08-2.78), however the 95\% CI was very close to 1 [20].

Fever, a non-specific symptom, is an important sign of UTI in infants, and together with pyuria leads to a urine culture being performed. However, somewhat surprisingly, pre-diagnosis fever was more prevalent in non-ESBL UTI children in our study. For afebrile children, a delayed diagnosis of UTI is unavoidable. In our study, the proportion of children aged $>2$ years was $43.2 \%$, and as noted, UTI in older children had a lower prevalence of fever than in infants <2 years. Overall 87 (26.9\%) UTI episodes had no fever. Even though ESBL was more common in afebrile children, being afebrile has limited application in clinical practice because there is no rationale for expecting or considering a diagnosis of UTI.

Recurrent UTI episodes were more likely to be ESBL-caused UTI than first UTI episodes $(\mathrm{OR}=2.7)$, a result consistent with two other studies, one from Turkey and one from Korea [11]. However, in the Korean study, CAKUT was also an independent risk factor for ESBL-caused UTI, a result in contrast to our study. In our study, a separate multivariate analysis (not shown) found that previous ESBL UTI increased the risk of ESBL UTI three-fold.

Serum creatinine levels were available in only 268 (83\%) of the episodes, but there was no significant difference in the prevalence of abnormally high levels between the ESBL and non-ESBL groups.

Any detected CAKUT had similar proportions for the ESBL and non-ESBL groups. Although our previous study found CAKUT to be a risk factor for recurrent UTI [21], in this current study recurrent UTI was a risk factor for ESBL UTI, but CAKUT was not an independent risk factor for ESBL UTI. 
Almost half of our UTI patients were aged more than 2 years, which, in our setting, may be due to the fact that parents of children with symptoms of UTI tend to delay seeking medical care for their children. Another possibility is that these parents may seek care for their child at another health facility prior to referral to our tertiary care center. Children who did not have an imaging study performed were older, possibly because their parents delayed seeking care or the original doctors followed the UTI investigation guidelines from developed countries which state that imaging studies are recommended as first-line investigations only in infants. And a final possibility is that the parents of older children may be less inclined to return for imaging investigations after their children have been discharged from hospital.

ESBL-caused UTI is serious in terms of antibiotic resistance since patients require parenteral antibiotic therapy and therefore admission to hospital, and diagnosis of ESBL UTI and treatment with appropriate antibiotics are usually delayed until the results of a urine culture are known. However, the results of our study indicate that the consequences of ESBL UTI such as urosepsis, CAKUT association and renal damage may not be as big a concern as originally thought. Recurrent UTI and being afebrile were the only factors associated with ESBL UTI, however, empirical antibiotics which are not sensitive to ESBL should still be considered even in recurrent UTI episodes until a urine culture positively indicates ESBL. A very important rule for reducing drug resistance is rational use of antibiotics and a strict antibiotic prescription policy.

Regarding ESBL infection, choice of antibiotic is an important issue, and careful prescription of antibiotics should therefore be made regarding local susceptibility patterns [22]. Apart from choice of antibiotics, the duration of treatment is also an issue, with the need to balance maximum effectiveness of therapy while minimizing the opportunity for the organisms to develop drug resistance. Our study found, $100 \%$ of ESBL organisms were sensitive to meropenem, imipenem and colistin while the sensitivity of ESBL organisms to amikacin and cefoxitin was $>80 \%$. Cefoxitin seems to be a promising antibiotic due to the low resistance rate, but it is not yet recommended for treatment of childhood UTI. In addition, its use is complicated because minimum inhibitory concentrations need to be monitored continually and doses adjusted accordingly [23], therefore the other four drugs are preferred. For non-ESBL-infected children, there are more choices as ESBL organisms were, in this study, found to have $<50 \%$ susceptibility to only two drugs (ampicillin and cotrimoxazole).

A study from Turkey in 53 children aged 3-12 years with amikacin-sensitive ESBL UTI [24] had a 96\% response rate with once daily intramuscular amikacin for 6 days (range 3-7 days). Overall, 30\% of the children had recurrent UTI, 30\% had antibiotic prophylaxis, and 90\% were given antibiotics in the last 3 months.

Our study was a retrospective study, thus histories of previous antibiotic and prophylactic therapy were not available; however, histories of antibiotic therapy are often unreliable because prescription of antibiotics in Thailand is not required by law and many parents obtain antibiotics from a drug store and they may not know the type of drug they receive. Whether the child completes the course of antibiotics is also not known. In our study, some children with VUR may have had antibiotic prophylaxis but there was no association between ESBL and VUR, thus we presume that antibiotic prophylaxis (co-trimoxazole) was unlikely to be related to ESBL UTI. The sample size was also too small to perform sub-group analyses. 
Amikacin can be a second-line drug but is highly limited to patients with amikacin-sensitive UTI and who are not able to be admitted for intravenous antibiotics since it can be injected intramuscularly once daily. However, it should be prescribed with caution since all aminoglycosides are ototoxic and nephrotoxic, particularly in patients with compromised renal function.

Administration of the appropriate antibiotic for ESBL is usually delayed due to the fact that empirical antibiotics for UTI are not sensitive to ESBL strains. Additionally, it takes 2-3 days for urine culture and sensitivity results reporting ESBL as a cause of UTI to become available. This is a serious concern because delayed diagnosis and/or treatment of UTI in children can cause significant morbidity including long-term renal consequences such as renal scarring, hypertension, and renal impairment in the worst cases [25, 26]. In this study, immediate consequences of ESBL UTI were not found, consistent with a report from Greece [8].

\section{Conclusions}

In our childhood UTI study, the prevalence of E. coli/K. pneumoniae ESBL dramatically increased from 2003-2008, and then plateaued for the next 6 years. Overall, ESBL was found in one-third of E. coli/K. pneumoniae UTI episodes. All ESBL strains were susceptible to carbapenem and colistin. Fortunately, ESBL UTI was not any more complicated than non-ESBL UTI and did not cause any immediate consequences. There was no clinical condition nor imaging study that could significantly predict ESBL. Previous UTI and being afebrile were the only two independent risk factors for ESBL UTI, and previous ESBL UTI was associated with increased risk of subsequent ESBL-caused UTI. No diagnostic factor could be identified for predicting ESBL UTI.

Abbreviations:

APD: anteroposterior mid-renal pelvic diameter

CAKUT: congenital anomalies of the kidney and urinary tract

DMSA: ${ }^{99 \mathrm{~m}} \mathrm{Tc}$ dimercaptosuccinic acid

E. coli: Escherichia coli

ESBL: extended-spectrum beta-lactamase

K. pneumoniae: $K$. pneumonia

RUS: renal ultrasound

UTI: urinary tract infection

VCUG: voiding cystourethrogram 


\section{References}

1. Sitthisarunkul N, Uthairat M, Dissaneewate P, McNeil E, Vachvanichsanong P. Characteristics and Findings of Childhood Urinary Tract Infection in the Last Decade. Urol Int 2019:1-6.

2. Tseng MH, Lo WT, Lin WJ, Teng CS, Chu ML, Wang CC. Changing trend in antimicrobial resistance of pediatric uropathogens in Taiwan. Pediatr Int 2008;50: 797-800.

3. Guidoni EB, Berezin EN, Nigro S, Santiago NA, Benini V, Toporovski J. Antibiotic resistance patterns of pediatric community-acquired urinary infections. Braz J Infect Dis 2008;12: 321-3.

4. Al Yousef SA, Younis S, Farrag E, Moussa H, Bayoumi FS, Ali AM. Clinical and Laboratory Profile of Urinary Tract Infections Associated with Extended Spectrum beta-Lactamase Producing Escherichia coli and Klebsiella pneumoniae. Ann Clin Lab Sci 2016;46:393-400.

5. Moore CE, Sona S, Poda S, et al. Antimicrobial susceptibility of uropathogens isolated from Cambodian children. Paediatr Int Child Health 2016;36:113-7.

6. Pitout JD, Laupland KB. Extended-spectrum beta-lactamase-producing Enterobacteriaceae: an emerging public-health concern. Lancet Infect Dis 2008;8:159-66.

7. Bradford PA. Extended-spectrum beta-lactamases in the 21st century: characterization, epidemiology, and detection of this important resistance threat. Clin Microbiol Rev 2001;14:933-51.

8. Tratselas A, Iosifidis E, Ioannidou M, et al. Outcome of urinary tract infections caused by extended spectrum beta-lactamase-producing Enterobacteriaceae in children. Pediatr Infect Dis J 2011;30:70710 .

9. Lee B, Kang SY, Kang HM, et al. Outcome of antimicrobial therapy of pediatric urinary tract infections caused by extended-spectrum beta-lactamase-producing Enterobacteriaceae. Infect Chemother 2013;45:415-21.

10. Ozcakar ZB, Yalcinkaya F, Kavaz A, et al. Urinary tract infections owing to ESBL-producing bacteria: microorganisms change--clinical pattern does not. Acta Paediatr 2011;100: e61-4.

11. Kim YH, Yang EM, Kim CJ. Urinary tract infection caused by community-acquired extendedspectrum beta-lactamase-producing bacteria in infants. J Pediatr (Rio J) 2017;93:260-6

12. Patwardhan V, Kumar D, Goel V, Singh S. Changing prevalence and antibiotic drug resistance pattern of pathogens seen in community-acquired pediatric urinary tract infections at a tertiary care hospital of North India. J Lab Physicians 2017;9:264-8.

13. Kocak M, Buyukkaragoz B, Celebi Tayfur A, et al. Causative pathogens and antibiotic resistance in children hospitalized for urinary tract infection. Pediatr Int 2016;58:467-71

14. van der Watt G, Omar F, Brink A, McCulloch M: Laboratory investigation of the child with suspected renal disease. In: Avner ED, Harmon WE, Niaudet P, Yoshikawa N, Emma F, Goldstein SL, eds. Pediatric Nephrology, 7th Edition. Heidelberg: Springer-Verlag, Berlin, 2016:613-636. 
15. Fernbach SK, Maizels M, Conway JJ. Ultrasound grading of hydronephrosis: introduction to the system used by the Society for Fetal Urology. Pediatr Radiol 1993;23:478-80.

16. Piepsz A, Colarinha P, Gordon I, et al. Guidelines for 99mTc-DMSA scintigraphy in children. Eur J Nucl Med 2001;28:BP37-41.

17. R Core Team (2018). R: A language and environment for statistical computing. R Foundation for Statistical Computing, Vienna, Austria. URL https://www.R-project.org.

18. Nisha KV, Veena SA, Rathika SD, et al. Antimicrobial susceptibility, risk factors and prevalence of bla cefotaximase, temoneira, and sulfhydryl variable genes among Escherichia coli in communityacquired pediatric urinary tract infection. J Lab Physicians 2017;9:156-62.

19. Amornchaicharoensuk Y. Clinical characteristics and antibiotic resistance pattern of pathogens in pediatric urinary tract infection. Southeast Asian J Trop Med Public Health 2016;47:976-82.

20. Kizilca O, Siraneci R, Yilmaz A, et al. Risk factors for community-acquired urinary tract infection caused by ESBL-producing bacteria in children. Pediatr Int 2012;54:858-62.

21. Vachvanichsanong $\mathrm{P}$, Dissaneewate $\mathrm{P}, \mathrm{McNeil}$ E. Childhood recurrent urinary tract infection in southern Thailand. Ren Fail 2013;35:66-71.

22. Haller M, Brandis M, Berner R. Antibiotic resistance of urinary tract pathogens and rationale for empirical intravenous therapy. Pediatr Nephrol 2004;19:982-6.

23. Guet-Revillet H, Emirian A, Groh M, et al. Pharmacological study of cefoxitin as an alternative antibiotic therapy to carbapenems in treatment of urinary tract infections due to extended-spectrumbeta-lactamase-producing Escherichia coli. Antimicrob Agents Chemother 2014;58:4899-901.

24. Polat M, Kara SS. Once-daily intramuscular amikacin for outpatient treatment of lower urinary tract infections caused by extended-spectrum beta-lactamase-producing Escherichia coli in children. Infect Drug Resist 2017;10:393-9.

25. Vachvanichsanong P. Urinary tract infection: one lingering effect of childhood kidney diseases-review of the literature. J Nephrol 2007;20:21-8

26. Beattie TJ, Mackenzie JR. Renal scarring after acute pyelonephritis. Arch Dis Child 1994;71:386-7. 


\section{Figure legends}

Figure 1. Number of children with E. coli/K. pneumoniae ESBL- and non-ESBL-caused urinary tract infections per year. The line represents the percentage of ESBL-caused urinary tract infections each year. (ESBL: extended-spectrum beta-lactamase).

Figure 2. Antibiotic resistance of bacterial isolates stratified by ESBL and non-ESBL urinary tract infections. 
Figures

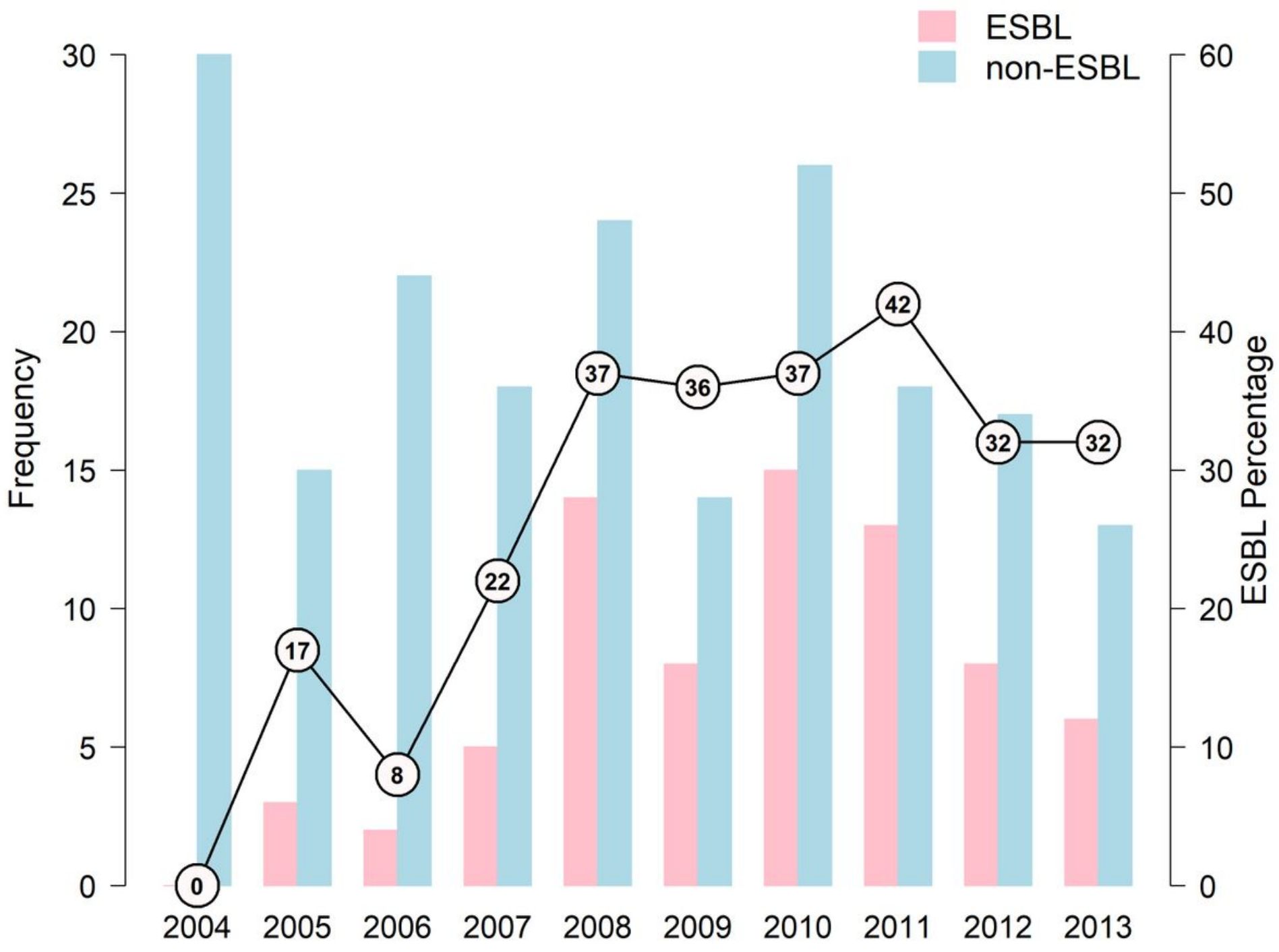

Figure 1

Number of children with E. coli/K. pneumoniae ESBL- and non-ESBL-caused urinary tract infections per year. The line represents the percentage of ESBL-caused urinary tract infections each year. (ESBL: extended-spectrum beta-lactamase). 


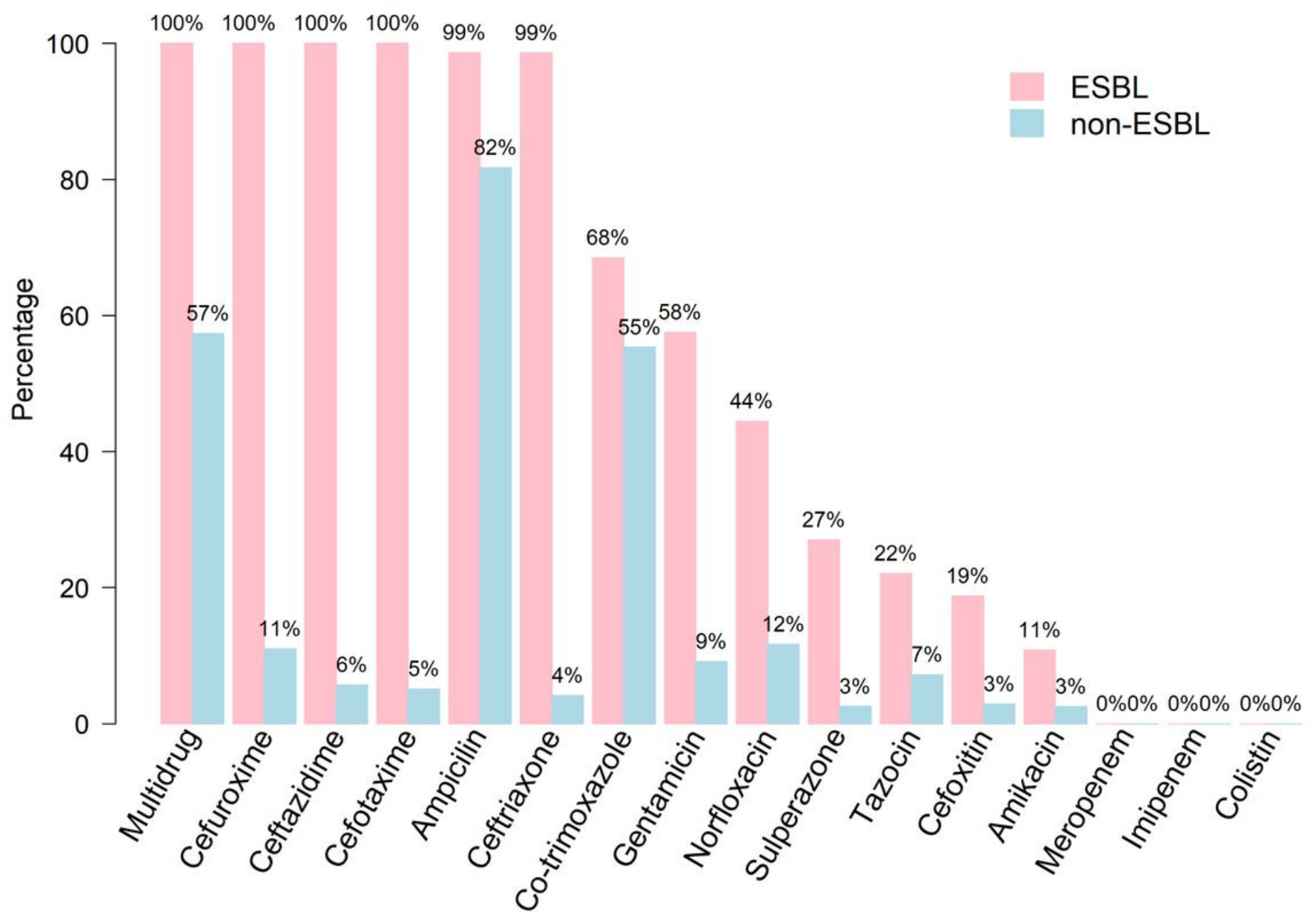

Figure 2

Antibiotic resistance of bacterial isolates stratified by ESBL and non-ESBL urinary tract infections.

\section{Supplementary Files}

This is a list of supplementary files associated with this preprint. Click to download.

- Table1.pdf

- Table2.pdf

- Table3.pdf 